\title{
Gravitational Description and Graphics of a Wormhole Structure-A Galactic Megamaximon
}

\author{
Yu. A. Khlestkov, N. Yu. Lukashina, M. Yu. Lukashin, P. Yu. Lukashin, N. S. Trushkin \\ National Research Nuclear University MEPhI, Moscow, Russia \\ Email: khlestkov@yandex.ru
}

How to cite this paper: Khlestkov, Yu.A., Lukashina, N.Yu., Lukashin, M.Yu., Lukashin, P.Yu. and Trushkin, N.S. (2019) Gravitational Description and Graphics of a Wormhole Structure-A Galactic Megamaximon. Journal of Modern Physics, 10 1299-1309.

https://doi.org/10.4236/jmp.2019.1011086

Received: September 10, 2019

Accepted: September 27, 2019

Published: September 30, 2019

Copyright $\odot 2019$ by author(s) and Scientific Research Publishing Inc. This work is licensed under the Creative Commons Attribution International License (CC BY 4.0).

http://creativecommons.org/licenses/by/4.0/

\begin{abstract}
Using the new exact solution of Einstein and Maxwell equations in the general relativity theory, they studied the internal structure of a geometric object with a nontrivial topology, the wormhole. They showed that the galactic black hole recently discovered by astronomers and astrophysicists as the part of the Event Horizon Telescope project with the radius about $10^{16} \mathrm{~cm}$ and the mass of about $10^{43} \mathrm{~g}$ can be a wormhole almost neutralized in charge with parameters close to critical-megamaximon, the radius of its neck curvature is equal to the so-called critical radius coinciding with half of the gravitational radius.
\end{abstract}

\section{Keywords}

Gravitation, Wormhole, Exact Solution, Megamaximon

\section{Introduction}

Gravitational interaction in cosmological scales (galaxies, universes) prevails. Great interest in this area is manifested in the study of objects with nontrivial properties-dark (exotic) matter, black holes, wormholes, etc. [1] [2]. This interest is increasing due to the recent discovery of such an object in deep space by astrophysicists (the Event Horizon Telescope project [3]).

This paper is devoted to the study of new exact particular solution of the Einstein and Maxwell equations use in the general relativity theory (GRT) [4] [5] [6] [7] to describe the internal structure and calculate the parameters of a compact stationary object-the wormhole within the scales of the galaxy and the universe. Previously, this method was applied to the microworld-to elementary particle and atomic nucleus lengths and showed good agreement with experimental results [7].

In this paper, we describe this solution, show the procedure of a wormhole occurrence analytically and graphically, and apply this model to study the possi- 
ble nature of a galactic object experimentally discovered and described in [3].

\section{Description of GRT Equations Particular Solution}

Using galactic lengths, let's consider the simplest spherically symmetric system of the neutral dust (the matter without pressure and temperature with zero entropy) with the energy density $\varepsilon_{s}$, with the energy-momentum tensor $\overbrace{T_{\mu \nu}}^{n_{u}}$ depending on 4-dust velocity $u_{\mu}$,

$$
{\stackrel{\sim}{T_{\mu v}}}_{s}^{s}=\varepsilon_{s} u_{\mu} u_{v},
$$

and from free electromagnetic field (without sources, i.e. with the electric charge density $\rho_{f}=0$ ), described by the electromagnetic field tensor $F_{\mu \nu}$ and energy-momentum tensor $T_{\mu}^{v}$ :

$$
\overbrace{T_{\mu}^{v}}^{f}=\frac{1}{4 \pi}\left(F_{\mu}^{\lambda} F_{\lambda}^{v}+\frac{1}{4} \delta_{\mu}^{v} F_{\alpha \beta} F^{\alpha \beta}\right),
$$

the interval square in which (metrics)

$$
\mathrm{d} s^{2}=g_{\mu \nu} \mathrm{d} x^{\mu} \mathrm{d} x^{v}
$$

in spherical coordinates

$$
x^{\mu}=\left\{x^{0}=\tau ; r ; \theta ; \varphi\right\}
$$

has the following form [8]:

$$
\mathrm{d} s^{2}=\mathrm{e}^{v(\tau, r)} \mathrm{d} \tau^{2}-\mathrm{e}^{\lambda(\tau, r)} \mathrm{d} r^{2}-R^{2}(\tau, r)\left(\mathrm{d} \theta^{2}+\sin ^{2} \theta \mathrm{d} \varphi^{2}\right),
$$

and described by Einstein's system of equations,

$$
G_{\mu \nu}=\kappa T_{\mu v}, \kappa=\frac{8 \pi k}{c^{4}},
$$

where $G_{\mu v}$ is Einstein's geometric tensor,

$$
T_{\mu v}=\overbrace{T_{\mu v}}^{s}+\overbrace{T_{\mu v}}^{f},
$$

and Maxwell's equations,

$$
F_{; v}^{\mu v}=0
$$

The system of Equations (1)-(8) in the associated with dust comoving frame of reference $u^{\mu}=\delta_{0}^{\mu} u^{0}$, where the energy-momentum tensor $T_{\mu v}$ of the internal space takes the following form:

$$
T_{\mu}^{v}=\operatorname{diag}\left(\varepsilon_{s}+\varepsilon_{f} ; \varepsilon_{f} ;-\varepsilon_{f} ;-\varepsilon_{f}\right),
$$

where $\varepsilon_{f}$ is the energy density of the electromagnetic field, and it looks like this $\left({ }^{\cdot}=\frac{\partial}{\partial \tau},{ }^{\prime}=\frac{\partial}{\partial r}\right)$ :

$$
\varepsilon=\frac{4 \pi R}{\kappa}\left(1-\mathrm{e}^{-\lambda} R^{\prime 2}+e^{-v} R^{\cdot 2}\right),
$$




$$
\begin{gathered}
\varepsilon^{\cdot}=4 \pi R^{2} R^{\cdot} \varepsilon_{f}, \varepsilon^{\prime}=4 \pi R^{2} R^{\prime}\left(\varepsilon_{s}+\varepsilon_{f}\right), \\
\varepsilon_{f}^{\cdot}=-4 \frac{R^{\cdot}}{R} \varepsilon_{f}, \\
\varepsilon_{f}^{\prime}=-4 \frac{R^{\prime}}{R} \varepsilon_{f}, \varepsilon_{s}^{\cdot}=-\frac{1}{2}\left(\lambda \cdot+\frac{R^{*}}{R}\right) \varepsilon_{s} .
\end{gathered}
$$

After the integration of the Equations (10)-(11) for the case of a system that is periodic in time and limited in space without relativistic rotation, we obtain the following particular solution of it [7]:

$$
\begin{gathered}
\varepsilon=\varepsilon_{g}(r)-\frac{Q^{2}}{2 R}, \\
\mathrm{e}^{v}=\mathrm{e}^{v_{\tau}(\tau)}, \\
\mathrm{e}^{\lambda}=\frac{R^{\prime 2}}{f^{2}(r)}, \\
\varepsilon_{f}=\frac{R_{c}^{2}}{\kappa R^{4}}, \\
f_{s}^{2}=\frac{R_{g}^{\prime}(r)}{\kappa R^{2} R^{\prime}}, \\
R=\frac{R_{g}}{2\left(1-f^{2}\right)}(1-\delta(r) \cos \eta), \\
\tau-\tau_{r}(r)=\frac{R_{g}}{2\left(1-f^{2}\right)^{\frac{3}{2}}}(\eta-\delta(r) \sin \eta) .
\end{gathered}
$$

Here $\varepsilon_{g}(r)$ is the total gravitational internal energy of the system on the radial coordinate $r$-an arbitrary function of $r$-the first integral of the system of differential Equations (10)-(11) of second order, $Q$ is the second first integral of this system, in this case the constant arising from its integration is electric system charge (which, we recall, was absent during the problem formulation-we considered the free electric field without sources. This means that the gravitational system generated electrical charge $Q$, whose geometric image in curved space will be clarified below.

$v_{\tau}(\tau)$ is an arbitrary function of time associated with the arbitrariness in the method of time coordinate $\tau$ measurement. Let $v_{\tau}=0$.

$f(r)$ - the third first integral of the system-an arbitrary function of $r$, in this case, $f^{2}<1$.

$R_{c}$ - the so-called critical radius of the system (10)-(11),

$$
R_{c}=\frac{\sqrt{k}}{c^{2}} Q .
$$

Gravitational radius proportional to the total gravitational mass of the system $M(r)=\varepsilon_{g}(r) / c^{2}:$

$$
R_{g}=\frac{2 k M(r)}{c^{2}},
$$


$\delta(r)$-the function of $r$, expressed through three independent first integrals of the Equations (10)-(11),

$$
\delta=\sqrt{1-\frac{4 R_{c}^{2}}{R_{g}^{2}}\left(1-f^{2}\right)},
$$

satisfying the following condition:

$$
0 \leq \delta(r)<1 .
$$

So, this system makes from uncharged dust and free radial electric field

$$
E_{r}=\sqrt{8 \pi \varepsilon_{f}}=\frac{Q}{R^{2}}
$$

(in the comoving frame of reference) an object-the electric charge $Q$. When $Q=0$, it passes into Tolman's well-known solution [8] -a system of dust-like neutral matter without an electromagnetic field. When $R_{g}=$ const, it passes into the well-known static vacuum world of Reissner-Nordstrom [9] of a point charge $Q$ with mass $M=$ const .

Singularity is a characteristic feature of Tolman and Reissner-Nordstemm's worlds, special cases of this solution

$$
R=0,-
$$

the point at which the curvature of space-time becomes infinite. This physically corresponds to the inversion of dust energy density (Tolman) or the energy density of the electromagnetic field (Reissner-Nordstrom) into infinity. The latter entails the most important feature-the Coulomb divergence of the point charge field.

This solution eliminates this singularity due to the formation of an object with a non-trivial topology—an unclosed wormhole.

\section{Wormhole Internal Structure and Parameters}

The appearance of a new wormhole in a curved space-time is due to inequality (17). Since $\delta(r)<1$, the solution (13) for $R(\tau, r)$-the radius of the 2-Gaussian curvature of the surface $\{\theta, \varphi\}$, at $R_{g}(r)>0$ shows that $R(\tau, r)$ does not vanish at any point:

$$
R(\tau, r)>0 .
$$

So, the electric field intensity (18) does not turn into infinity at any point.

$$
E_{r}(\tau, r) \neq \infty .
$$

Thus, the Coulomb divergence of the point charge field, which is present in all models within the special relativity theory (SRT), i.e. in flat Minkowski space, is eliminated.

Figure 1, Figure 2 show how the wormhole is formed. The family of coordinate surfaces (spheres $=R=$ const and cones $\theta=$ const) in a flat space has the feature (singularity) $R=0$. If $R(\tau, r) \neq 0$, then the family of cones turns into the family of surfaces of hyperbolic type with negative Gaussian curvature, 


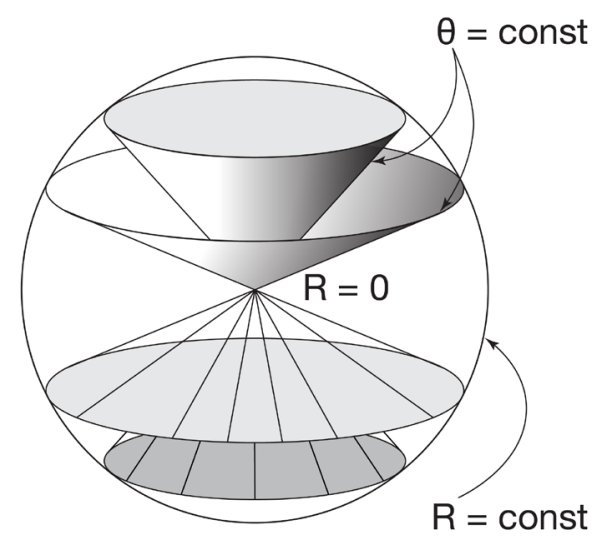

Figure 1 . The family of coordinate surfaces $R=$ const and $\theta=$ const in the empty flat space of SRT.

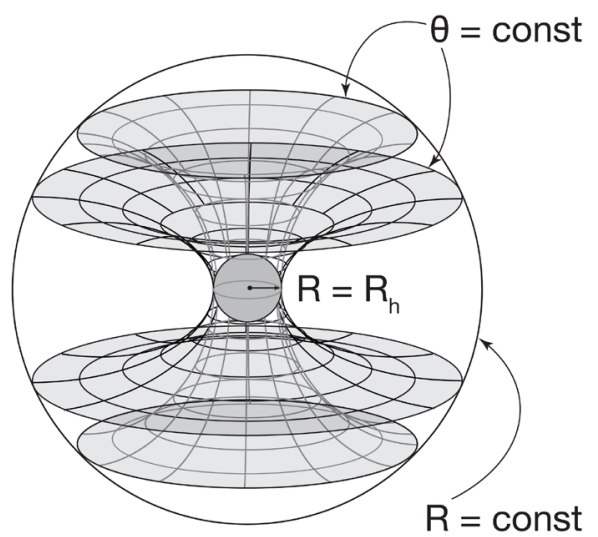

Figure 2. The family of coordinate surfaces $R=$ const and $\theta=$ const in the curve space of GRT in the world with a constant charge $Q=$ const. The neck radius makes $R_{h}$.

similar to one-cavity hyperboloid, whose main radii lie on opposite sides of these 2 -surfaces, that is, they have different signs (Figure 2). This is a wormhole with a neck (horn, throat) - the sphere of an extreme radius $R_{h}=$ const .

According to the solution (13), this wormhole has extreme static surfaces-the necks with a constant radius of 2-Gaussian curvature $R_{h}$, determined from the following condition:

$$
r=r_{h}, R_{h}^{*}=0,
$$

which is always equal to doubled classical radius $R_{f}$ if we substitute the conditions (22) into the hole Equation (13):

$$
\begin{gathered}
R_{f}(r)=\frac{Q^{2}}{2 M c^{2}}=\frac{R_{c}^{2}}{R_{g}}, \\
R_{h}=2 R_{f h}=\xi R_{c}=\frac{R_{g h}}{2} \xi^{2},
\end{gathered}
$$

where $\xi$ is the ratio of the electric charge $Q$ to the gravitational "charge" 
$\sqrt{k} M_{h}$ at the neck:

$$
\xi=\frac{Q}{M_{h} \sqrt{k}} .
$$

We will solve the Cauchy problem with the initial conditions. Let the radius of curvature $R(0, r)=R(0)$ will be a periodic function of the radial coordinate $r$ at $\tau=0 \quad(\eta=\pi)$ :

$$
R(0)=\frac{R_{\max }}{2}+\frac{R_{h}}{2}-\frac{R_{\max }-R_{h}}{2} \cos 2 \chi,
$$

where $\chi=\pi \frac{r}{r_{h}}, R_{\max }$ is the radius $R$ at $\chi=\frac{\pi}{2}$ in the state of maximum expansion of the wormhole's internal space. Let us set the initial change rate of this radius, for example:

$$
R^{\cdot}(0, r)=R^{\cdot}(0)=0,
$$

and let the dust energy density is homogeneous in the initial state for simplicity:

$$
\varepsilon_{s}(0)=\varepsilon_{s h}=\text { const }
$$

where $\varepsilon_{s h}$ is the dust energy density on the wormhole neck.

The initial data (25)-(27) allows to express all functions of $r$ and constants through the dimensionless parameter $\xi=\frac{R_{h}}{R_{c}}=\frac{Q}{\sqrt{k} M_{h}}$, the critical radius $R_{c}$ and $R(0)$ :

$$
\begin{gathered}
R_{g}=R_{g h}\left(\frac{R(0)}{R_{h}}\right)^{3}, \\
R_{g h}=\frac{2 R_{c}}{\xi}, \\
\varepsilon_{s h}=\frac{6}{\kappa R_{c}^{2}} \frac{1}{\xi^{4}}, \\
1-f^{2}=\frac{R_{g}}{R(0)}-\frac{R_{c}^{2}}{R^{2}(0)}, \\
1-f_{h}^{2}=\frac{1}{\xi^{2}}, \\
\delta=1-\frac{2 R_{c}^{2}}{R_{g} R(0)}, \\
\delta_{h}=0, \\
R_{\max }=\frac{1}{2} R_{c} \xi^{2} \sqrt{1+\sqrt{1+\frac{8}{\xi^{4}}}}, \\
R_{\min }=2 R_{c} \frac{1}{\xi^{2}} \frac{1}{\sqrt{1+\sqrt{1+\frac{8}{\xi^{4}}}}}, \\
R_{\max } R_{\min }=R_{c}^{2} .
\end{gathered}
$$


A geometric object whose $\xi=1$, that is, the electric charge $Q$ is equal to the gravitational $\sqrt{k} M_{h}$ is called a maximon. It has extreme parameters:

$$
\begin{gathered}
R_{h}=R_{c}=2 R_{f h}=\frac{R_{g h}}{2}, R_{\max }=R_{\min }=R_{c}, \\
\varepsilon_{s}(0)=\varepsilon_{s c}=\frac{6}{\kappa R_{c}^{2}} .
\end{gathered}
$$

Thus, this simplest solution of the GTR equations describes the world that is periodic in time and space, formed by a free radial electric field and a neutral dust-like substance, which is a pulsating unclosed wormhole geometrically, which is an electric charge $Q$ itself generated by a curved space changing its sign through the spatial period $r_{h}(\chi \in[0, \pi])$, like the electric field $E_{r}$ (Figure 3).

This tunnel ("well") can be cut off over a period along $r$ and glued to the vacuum static world of Reissner-Nordstrom [9] through two static necks-an external solution for the field of the point charge $Q$ of mass $M_{h}$-asymptotically flat in vacuum at infinity (Figure 4).

\section{Universality of Gravitational Interaction}

Traditionally, the gravitational interaction is considered to be vanishingly small as compared to both electromagnetic and nuclear one in the microworld, but it prevails in the megaworld, at the lengths of galaxies and universes. However, this is not quite true. Gravitational interaction is universal. The first argument is in plain sight. The internal structure of the proton was detected experimentally: its charge radius, as measured by the Lamb shift on muon hydrogen, turned out to be equal to $0.8409 \times 10^{-13} \mathrm{~cm}$ [10]. This is already enough to consider space-time as curved, i.e. formed by a gravitational field.

The logic is simple. If it were flat (with the Riemann-Christoffel curvature tensor identically equal to zero), then it would be necessary to be empty, according to the Einstein equations, $G_{\mu \nu}=\kappa T_{\mu \nu}$ : if $G_{\mu \nu} \equiv 0$, then the matter energy-momentum tensor is identically zero, $T_{\mu \nu} \equiv 0$. So the space would be empty. This also means that if there can be material objects in it, then they must be the point features of the field that do not belong to Riemannian space itself, including proton, and any other particles. And this is contrary to the experiment. Therefore, the solution of the Einstein and Maxwell equations given in this paper is applicable at any lengths.

This conclusion confirms the Einstein equations in General relativity: they do not contain a fundamental constant of dimension of length (of the two constants $c$ and $k$ it is impossible to construct such a value).

So, being applied to a proton, it gives the value of its neck radius $R_{h}$, equal to $0.8412 \times 10^{-13} \mathrm{~cm}$ [7]. This coincides with its experimental value $0.8409 \times 10^{-13}$ $\mathrm{cm}[7]$ within the accuracy of $0.04 \%$.

Thus, for the first time, we obtain reliable experimental confirmation of GRT in the relativistic region of the microworld, in which the influence of space-time curvature, i.e. gravitational field, was considered negligible. 


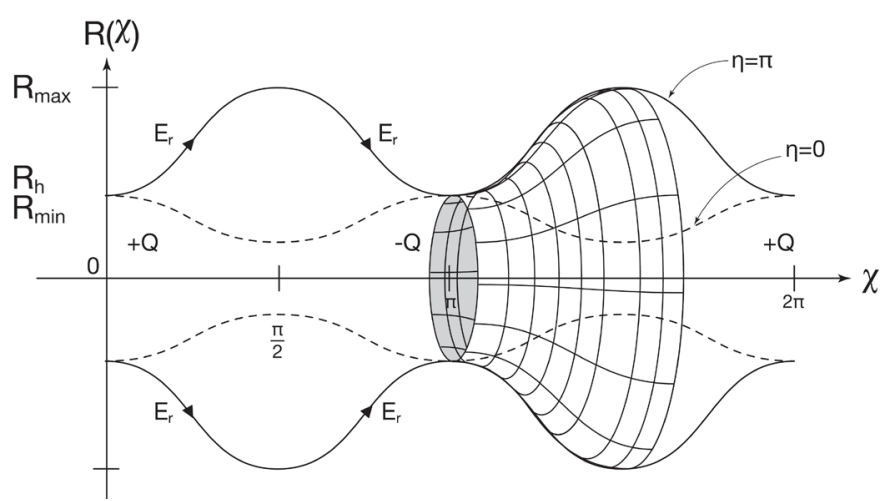

Figure 3. The internal space of the electric charge $Q$ (wormhole)-the tunnel that does not close in time and in space in the state of maximum $(\eta=\pi)$ expansion and maximum compression $(\eta=0)$. The cyclic coordinate $\varphi$ is directed orthogonally to the plane of the figure. The cyclic coordinate $\theta$ is not shown.

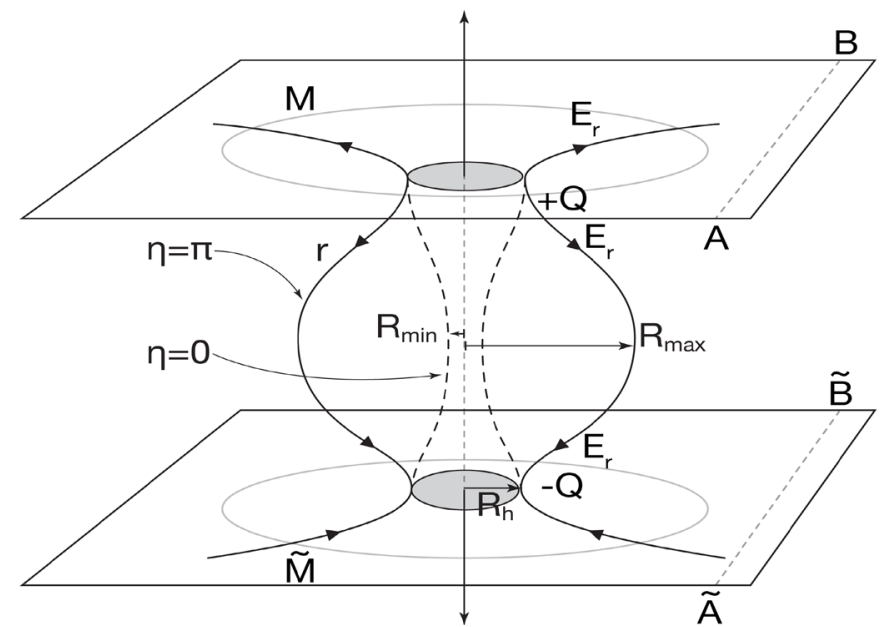

Figure 4. A wormhole pulsating from the state of maximum expansion $(\eta=\pi)$ to the state of maximum compression $(\eta=0)$, cut off and glued on the necks within the period with two parallel asymptotically flat worlds- $M$ and $\tilde{M}$, a geometric image of an electric charge $Q$-pair particle-antiparticle. The coordinate $r$ is directed by arrows, the cyclic coordinate $\varphi$ is orthogonal to the picture, the cyclic coordinate $\theta$ is not shown. If you cut the worlds $M$ and $\tilde{M}$ along dashed lines $A B$ and $\widetilde{A B}$ and glue them together, you will get one space with two holes (Wheeler's handle).

\section{Galactic Megamaximon}

In this paper, we apply the solution of GRT Equations (10)-(11) to the megaworld: let's show that an object found in the constellation Virgo with the radius $R_{h}$ of about $10^{16} \mathrm{~cm}$ and the solar mass of about $6.5 \times 10^{9}$ mass of the Sun that was discovered by astrophysicists and astronomers within the Event Horizon Telescope project can be an extreme wormhole-megamaximon (i.e. it has $\xi=1)$ 
According to (23), the radius of the wormhole neck curvature makes.

$$
R_{h}=2 R_{f h}=\frac{Q^{2}}{M_{h} c^{2}} .
$$

Let express the electric charge $Q$ through the fundamental charge $e$ and the number of such uncompensated charges on the neck $N_{Q}$ :

$$
Q=e N_{Q}=e N_{p}\left(1-\eta_{e}\right) \text {, }
$$

where $N_{p}$ is the number of protons in the nuclei of atoms, $\eta_{e}=\left|1-\frac{N_{e}}{N_{p}}\right|$ is the charge neutralization factor, $N_{e}$ is the number of electrons in the neck atoms.

The mass of the neck is estimated by the number of nucleons in the nuclei:

$$
M_{h}=m_{p} N_{p}\left(1+\eta_{n}\right) \text {, }
$$

where $\eta_{n}=\frac{N_{n}}{N_{p}}$ is the neutronization coefficient of nuclei.

Substituting (30) and (31) in (23), we obtain the following for the hole radius:

$$
R_{h}=\frac{e^{2}}{m_{p} c^{2}} N_{p} \cdot \frac{\left(1-\eta_{e}\right)^{2}}{\left(1+\eta_{n}\right)} .
$$

From the calculation of the proton parameters as a wormhole [7] it follows that

$$
\frac{e^{2}}{m_{p} c^{2}}=\frac{r_{h p *}}{\gamma_{* p}},
$$

where $r_{h p *}$ is the radius of the proton neck, taking into account the spin, $\gamma_{* p}$ is the Lorentz factor of the proton on its neck.

Substituting (33) in (32), we get the following:

$$
R_{h}=\frac{r_{h p^{*}}}{\gamma_{* p}} N_{p} \frac{\left(1-\eta_{e}\right)^{2}}{\left(1+\eta_{n}\right)} .
$$

from this, having entered the mass of the mega-wormhole neck $M_{h}$ from (31), we have for the coefficient of neutralization the following:

$$
\left|1-\eta_{e}\right|=\sqrt{\frac{R_{h} \gamma_{* p}}{r_{h p *}} \frac{m_{p}\left(1+\eta_{n}\right)^{2}}{M_{h}}} .
$$

Substituting the numerical values of the its quantities in (35):

$$
\begin{aligned}
& m_{p}=1.6726 \times 10^{-24} \mathrm{~g}, \\
& r_{h * p}=0.8412 \times 10^{-13} \mathrm{~cm}, \\
& R_{h}=10^{16} \mathrm{~cm}, \\
& M_{h}=5.6 \times 10^{9} \times 1.9885 \times 10^{33} \mathrm{~g}, \\
& \gamma_{* p}=0.548 \times 10^{3}, \\
& \eta_{n} \approx 1,
\end{aligned}
$$




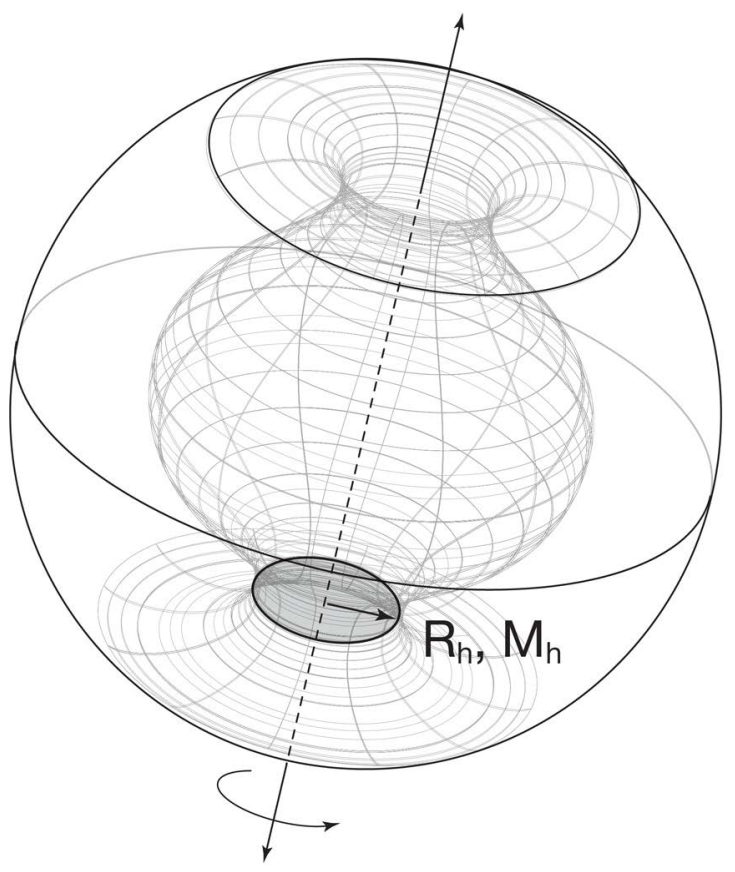

Figure 5. "Wandering" galactic wormhole with extreme radius $R_{h}$ and the neck mass $M_{h}$ (megamaximon).

we obtain the following:

$$
\left|1-\eta_{e}\right| \approx 0.63 \times 10^{-17} \ll 1 .
$$

This means that this wormhole is almost neutralized by charge: $\eta_{e} \rightarrow 1$.

Let's estimate the value of the dimensionless parameter $\xi$ :

$$
\xi=\frac{R_{h}}{R_{c}}, R_{c}=\frac{Q \sqrt{k}}{c^{2}}=\frac{e \sqrt{k}}{c^{2}} N_{p}\left|1-\eta_{e}\right|, \quad \xi=\frac{r_{h p *}\left|1-\eta_{e}\right|}{\gamma_{* p}\left(1+\eta_{n}\right) r_{c}} .
$$

$r_{c}=1.3807 \times 10^{-34} \mathrm{~cm} \mathrm{[7].}$

Substituting the values of the parameters in (37), we obtain the following for $\xi$ :

$$
\xi=\frac{0.8412 \times 10^{-13} \times 0.3 \times 10^{-17}}{1.3807 \times 10^{-34} \times 2 \times 0.548 \times 10^{3}} \approx 3.5 \rightarrow 1 .
$$

Thus, the object discovered by astronomers and astrophysicists in the galaxy is the wormhole, the practical maximum wormhole by parameters, the megamaximon.

Considering that it is geometrically similar to a torus on the photo, it can be assumed that this is the wormhole wandering between galaxies, obtained from the solution (13) by gluing through two necks with two Reissner-Nordstrom vacuum worlds, which are cut and glued along the circles of radius, much larger radius of the neck (Figure 5).

\section{Summary}

The paper describes the solution of general relativity equations for spherically 
symmetric dust-like matter and the radial electric field and shows analytically and graphically that it describes a space object of nontrivial topology-the wormhole, with its two necks extending into two parallel vacuum asymptotically flat worlds or into one world if these surfaces are cut and glued accordingly.

This model is used to describe the newly discovered galactic black hole (presumably) with a radius of about $10^{16} \mathrm{~cm}$ and a mass of $10^{43} \mathrm{~g}$ by astrophysicists and astronomers in Virgo constellation within the Event Horizon Telescope project. It is shown that it can be a hole (do not confuse it with a Black Hole) in space-time-the wormhole that is almost compensated by electric charge with the radius equal to the critical $R_{c}$, and equal to half of the gravitational radius $R_{g}$. That is, this object is megamaximon.

\section{Acknowledgements}

We express our gratitude to S.I. Okov for his help in translating the article.

\section{Conflicts of Interest}

The authors declare no conflicts of interest regarding the publication of this paper.

\section{References}

[1] Visser, M. (1996) Lorentzian Wormholes. Springer Verlag, Berlin.

[2] Bronnikov, K.A., Fabris, J.C. and Rodrigues, D.C. (2015) International Journal of Modern Physics D, 25, Article ID: 1641005. https://doi.org/10.1142/S0218271816410054

[3] Akiyama, K., Alberdi, A, Alef, W., Asada, K., Azulay, R., Baczko, A.-K., Ball, D. and Volokovic, M. (2019) The Astrophysical Journal Letters, 875, L4.

[4] Khlestkov, Yu.A. (2005) Journal of Experimental and Theoretical Physics, 101, 259. https://doi.org/10.1134/1.2047791

[5] Khlestkov, Y.A. and Sukhanova, L.A. (2016) Foundation Physics, 46, 668-688. https://doi.org/10.1007/s10701-016-9998-6

[6] Khlestkov, Yu.A. and Sukhanova, L.A. (2017) Chinese Journal of Physics, 55, 1794-1807. https://doi.org/10.1016/j.cjph.2017.08.007

[7] Khlestkov, A.Yu. and Khlestkov, Yu.A. (2019) Russian Physics Journal, 62, 264-283. https://doi.org/10.1007/s11182-019-01709-9

[8] Landau, L.D. and Lifshitz, E.M. (1975) The Course of Theoretical Physics. Vol. 2. The Classical Theory of Fields. Butterworth-Heinemann, London.

[9] Hawking, S.W. and Ellis, G.F.R. (1975) The Large Scale Structure of Space-Time. Cambridge University Press, Cambridge.

[10] Pole, R., et al. (2010) Nature, 466, 213. 